\title{
Differential Cross Sections for Neutron Elastic and Inelastic Scattering on ${ }^{23} \mathrm{Na}$
}

\author{
J.R. Vanhoy ${ }^{1,3,4}$ a, S.F. Hicks ${ }^{2}$, A. Chakraborty ${ }^{3,4}$, B.R. Champine ${ }^{5}$, B. Combs ${ }^{2}$, B.P. Crider ${ }^{4}$, L.J. \\ Kersting $^{2}$, A. Kumar ${ }^{3,4}$, C.J. Lueck ${ }^{2}$, P.J. McDonough ${ }^{2}$, M.T. McEllistrem ${ }^{3,4}$, E.E. Peters ${ }^{3}$, F.M. \\ Prados-Estévez ${ }^{3,4}$, L. Sidwell ${ }^{2}$, A. Sigillito ${ }^{2}$, D.W. Watts ${ }^{1}$, and S.W. Yates ${ }^{3,4}$ \\ ${ }^{1}$ Dept. of Physics, United States Naval Academy, Annapolis, MD 21402, USA \\ ${ }^{2}$ Dept. of Physics, University of Dallas, Irving, TX 75062, USA \\ ${ }^{3}$ Dept. of Chemistry, University of Kentucky, Lexington, KY 40506, USA \\ ${ }^{4}$ Dept. of Physics \& Astronomy, University of Kentucky, Lexington, KY 40506, USA \\ ${ }^{5}$ Dept. of Physics \& Nuclear Engineering, U.S. Military Academy, West Point, NY 10996, USA
}

\begin{abstract}
Measurements of neutron elastic and inelastic scattering from ${ }^{23} \mathrm{Na}$ have been performed for sixteen incident neutron energies above $1.5 \mathrm{MeV}$ with the 7-MV University of Kentucky Accelerator using the ${ }^{3} \mathrm{H}(\mathrm{p}, \mathrm{n})$ reaction as the neutron source. These measurements were complemented by $\gamma$-ray excitation functions using the $\left(n, n^{\prime} \gamma\right)$ reaction. The time-of-flight technique is employed for background reduction in both neutron and $\gamma$ ray measurements and for determining the energy of the scattered neutrons. Cross section determinations support fuel cycle and structural materials research and development. Previous reaction model evaluations [1] relied primarily on total cross sections and four $\left(\mathrm{n}, \mathrm{n}_{0}\right)$ and $\left(\mathrm{n}, \mathrm{n}_{1}\right)$ angular distributions in the $\mathrm{E}_{\mathrm{n}}=5$ to $9 \mathrm{MeV}$ range. The inclusion of more inelastic channels at lower neutron energies provides additional information on direct couplings between elastic and inelastic scattering as a function of angular momentum transfer. Reaction model calculations examining direct collective and statistical properties were performed.
\end{abstract}

\section{Introduction}

The USDOE Advanced Fuels program funds R\&D on innovative next generation light water reactors and future fast systems. High-quality neutron scattering data guide sensitivity analyses of fuel performance during irradiations, including the influence of surrounding coolants and structural materials. Recent evaluations of the coolant ${ }^{23} \mathrm{Na}$ revealed there are significant discrepancies in cross sections between the evaluated nuclear libraries, especially above $1 \mathrm{MeV}$ where there is a critical lack of measured data.

Differential scattering cross sections guide nuclear reaction mechanism calculations in the few$\mathrm{MeV}$ fast-neutron region. Model calculations are difficult in ${ }^{23} \mathrm{Na}$ due to the overlapping resonance structure and coupling to direct processes. Sodium-23 is strongly deformed and the effect must be included. Cross sections must therefore be measured. Elastic scattering data guide optical model

${ }^{\text {a } C o r r e s p o n d i n g ~ a u t h o r: ~ v a n h o y @ u s n a . e d u ~}$

This is an Open Access article distributed under the terms of the Creative Commons Attribution License 2.0, which permits unrestricted use, distribution, and reproduction in any medium, provided the original work is properly cited. 
treatments while inelastic differential cross sections provide the crucial information on the coupling to the direct reaction component.

\section{Measurements}

The differential scattering cross sections were measured as a function of angle using bunched beams from the University of Kentucky accelerator with the ${ }^{3} \mathrm{H}(\mathrm{p}, \mathrm{n})$ reaction as a neutron source. The shielded neutron detector was mounted at distances of 2.2 to $3.8 \mathrm{~m}$ from the scattering samples, which were hung $7.0 \mathrm{~cm}$ from the center of the tritium-containing gas cell. The FWHM energy resolution was approximately $80 \mathrm{keV}$. Neutrons were detected in a $11.5 \mathrm{~cm}$ diameter x $2.5 \mathrm{~cm}$ thick $\mathrm{C}_{6} \mathrm{D}_{6}$ liquid scintillator, which permitted pulse-shape rejection of $\gamma$-ray events. Measurements were made at $10^{\circ}$ intervals from $30^{\circ}$ to $150^{\circ}$. The scattering yields were extracted from the TOF spectra using a peak fitting program that models each peak with two Gaussians and an exponential tail and incorporates kinematic constraints into peak positions and peak shapes. Yields were corrected for neutron attenuation and multiple scattering in the sample using Monte Carlo methods. Absolute normalization of the cross sections were determined by measuring n-p scattering [2,3]. Representative (n,n) angular distributions are shown in Sec. 3 below.

Gamma-rays were detected using a Compton-suppressed n-type HPGe detector with 51\% relative efficiency and an energy resolution of $2.1 \mathrm{keV}$ FWHM at $1.33 \mathrm{MeV}$. Compton suppression was achieved using a BGO annular detector surrounding the HPGe detector. The neutron scattering facilities, TOF neutron background suppression, neutron monitoring and data reduction techniques have been described elsewhere [4].

Gamma-ray excitation functions were measured for incident neutron energies between 1.5 and 4.0 MeV. Yields from the measurements were corrected for $\gamma$-ray detection efficiency and were normalized to yields from a "long counter" neutron monitor which were corrected for efficiency as a function of neutron energy in order to obtain relative $\gamma$-ray production cross sections. The absolute cross section normalization was obtained by comparison to ${ }^{56} \mathrm{Fe}$ cross sections, appropriately energyaveraged [5]. The $\left(n, n^{\prime}\right)$ cross section to a given level can be derived from the $\gamma$-ray cross sections by considering the balance between depopulating and feeding transitions. Representative neutron inelastic scattering cross sections derived from $\gamma$-ray production cross sections are shown in Fig. 1.

Inelastic scattering cross sections to a given final state can be measured directly via the (n,n') reaction or derived from $\gamma$-ray excitation functions. Angular distributions of the $\left(n, n^{\prime}\right)$ reaction provide the best information on the reaction mechanism while $\gamma$-ray measurements provide the best information on the partition of reaction strength.

\section{Conclusions}

Angle-integrated elastic ${ }^{23} \mathrm{Na}\left(\mathrm{n}, \mathrm{n}_{0}\right)$ cross sections agree with both the ENDF/B-VII.1 [6] and JENDL-4.0 [7] evaluations rather well while the JEFF-3.1.2 [8] evaluation is significantly higher. Elastic scattering results are compared to the ENDF database and a literature optical model calculation in Fig. 1. Inelastic scattering cross sections from the ENDF library tend to be larger than our experimental data by $\sim 15 \%$, while cross sections from the JEFF library tend to be smaller by $\sim 15 \%$. Inelastic scattering results are shown in Fig. 2 compared to the JENDL database and a literature optical model calculation. 


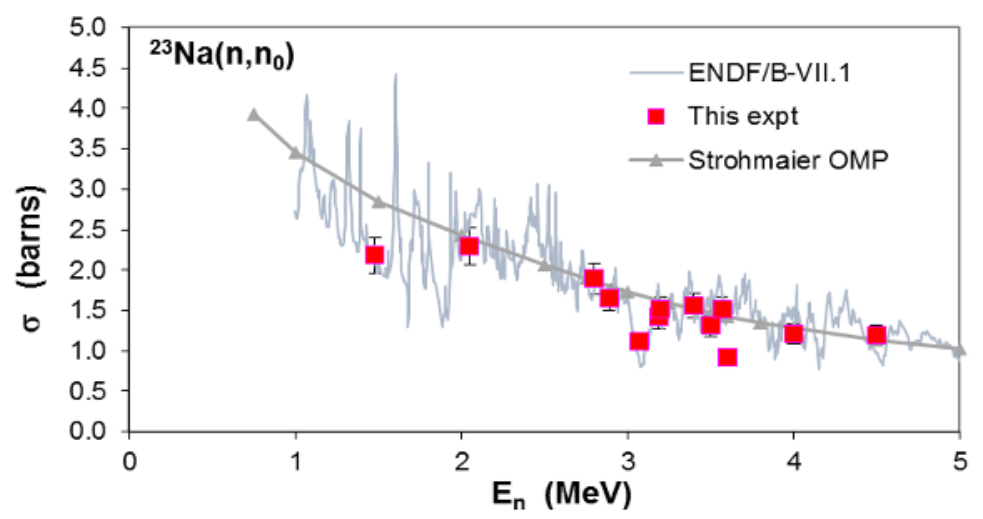

Figure 1. Comparison of measured elastic angle-integrated cross sections with ENDF database and the coupled channels treatment by Strohmaier [1]. Measured data are consistent with the ENDF database. The agreement with JENDL database (not shown) is equally well.

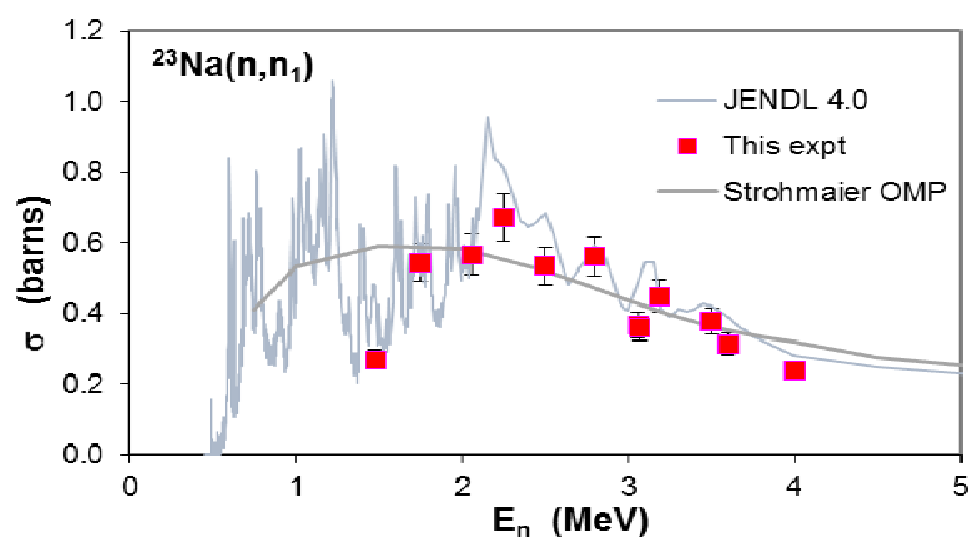

Figure 2. Comparison of the measured angle-integrated cross sections to the 440-keV level with the JENDL database and the coupled channels treatment by Strohmaier [1].

Nuclear structure interpretations of ${ }^{23} \mathrm{Na}$ are rather limited. The most recent discussion is that of Durell et al. [9] which indicates that significant deformation is present in this mass region. The ${ }^{23} \mathrm{Na}$ level scheme is generally discussed as rotational with strong core-excitation components [9]. The large ${ }^{23} \mathrm{Na}$ deformation impacts the differential cross sections.

A reaction mechanism interpretation of the ${ }^{23} \mathrm{Na}$ cross section in the 1 to $4 \mathrm{MeV}$ range is rather difficult. This range is above the isolated resonance region but not yet smoothly varying. Differential cross sections are comprised of the compound nucleus and direct reaction mechanisms; however the compound nuclear portion may be influenced by isolated resonance effects not addressable within the optical model treatment. This is certainly the case for inelastic scattering to the second and higher inelastic levels, where the resonances are clearly resolved [10].

We have investigated the applicability of the coupled channels code ECIS06 [11] to our $\mathrm{E}_{\mathrm{n}}>3.6$ $\mathrm{MeV}$ energy $\left(\mathrm{n}, \mathrm{n}_{0}\right)$ and $\left(\mathrm{n}, \mathrm{n}_{1}\right)$ data sets. Optical model and coupling parameters were taken from Strohmaier [1] who examined $E_{n}>5 \mathrm{MeV}(n, x)$ data for the purposes of applying the results to radiation dosimetry. Our analysis provides information on the deviations that can be anticipated from the coupled-channels reaction model predictions in the $\mathrm{E}_{\mathrm{n}}=2$ to $5 \mathrm{MeV}$ region. 

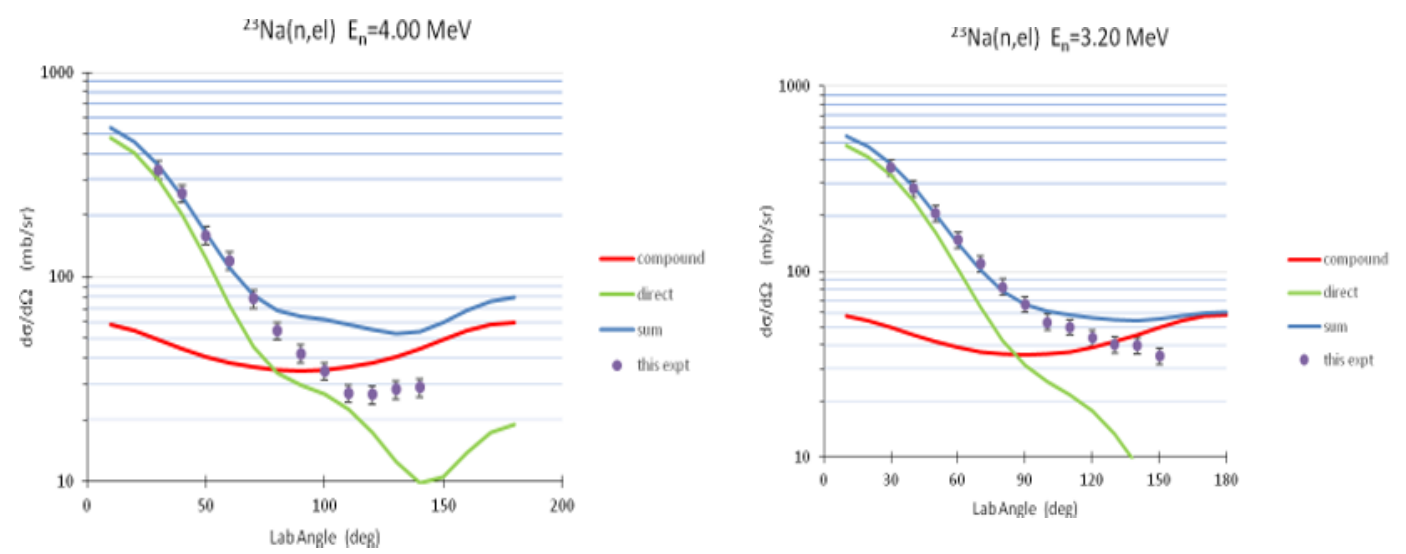

Figure 3. Coupled channels calculations [1] for elastic scattering compared to data. Large-angle data indicate the compound elastic contribution is too large.

In general (and illustrated in Fig. 3), we find that the Strohmaier parameters [1] provide an adequate description of the forward-angle elastic differential cross section. The compound elastic process, apparent at angles $>90^{\circ}$, is too large in that calculation, indicating the imaginary potential, $\mathrm{W}_{\mathrm{D}}$, should be substantially reduced. Dispersive optical model calculations in this mass region also point to smaller absorption at lower energies [12].

Although not shown in this manuscript, the shapes of the $\left(n, n_{1}\right)$ and $\left(n, n_{2}\right)$ differential cross sections for scattering to the $440-\mathrm{keV}$ and $2076-\mathrm{keV}$ levels, indicate the presence of direct coupling. This is a result of the strong deformations known in the neighbouring nuclei in this mass region [13] which force inclusion of direct processes into descriptions of the inelastic channels at lower energies than is typical with coupled channel treatments.

\section{Acknowledgements}

Research at the University of Kentucky Accelerator Laboratory is supported by a contract from the U.S. Department of Energy-Nuclear Energy Universities Program, the U.S. National Science Foundation grants program, the USMA Nuclear Science and Engineering Research Center, the USNA James Kinnear Fellowship, and the Cowan Physics Fund at the University of Dallas.

\section{References}

1. B. Strohmaier, Ann. Nucl. Energy 20, 533 (1993).

2. A.D. Carlson et al., Nucl. Data Sheets 110, 3215 (2009).

3. G.M. Hale, "ENDF/V-II.1 ${ }^{1} \mathrm{H}(\mathrm{n}, \mathrm{n})$ Reaction Evaluation", Evaluation of Oct 2005, Distribution of Dec 2006. Available at http://www.nndc.bnl.gov/sigma/ .

4. P.E. Garrett, N. Warr, and S.W. Yates, J. Res. Natl. Inst. Stand. Technol. 105, 141 (2000).

5. M.B. Chadwick, P.G. Young, C.Y. Fu, ENDF/B-VII.1 Data Evaluation for ${ }^{56} \mathrm{Fe}$, Sept 1996.

6. D.C. Larson et al., ENDF/B-VII.1 Data Evaluation for ${ }^{23} \mathrm{Na} 1977$, and updates through Dec 2011.

7. K. Shibata et al., JENDL Data Evaluation for ${ }^{23} \mathrm{Na}$, and updates through Mar 2002.

8. E. Fort et al., JEFF-3.1.2 Data Evaluation for ${ }^{23} \mathrm{Na}$, and updates through May 2005, and A. Santamarina et al., JEFF-3.1.1 Nucl. Data Library, Report OECD/NEA JEFF Report 22, 2009.

9. J.L. Durell et al., J.Phys. A 5, 302, (1972).

10. C. Rouki et al., Nucl. Instrum. Meth. A 672, 82 (2012).

11. J. Raynal, computer code NEA-0850/18 ECIS-06.

12. M.M. Nagadi, et al., Phys. Rev C 68, 044610 (2003).

13. R. Capote et al., Nucl. Data Sheets 110, 3107 (2009). Online at http://www-nds.iaea.org/RIPL-3/ 